\title{
Appropriate design of structural membranes
}

\author{
Josep Llorens \\ Barcelona School of Architecture (ETSAB), Universitat Politècnica de Catalunya \\ Diagonal, 649 - 08028 Barcelona, Spain \\ E-mail: ignasi.llorens@upc.edu \\ web page: https://wwwgat.webs.upc.edu/portada.html
}

\begin{abstract}
"Structural membranes, if not designed as such, require an imposing steel structure" (J.Hennicke).

Progress is being made in the understanding of the appropriateness of membrane structures that are much more efficient than bending solutions based on trusses and cantilevers. But many designs do not take it into account to the point that membrane structures frequently end up being covered conventional steel structures.
\end{abstract}

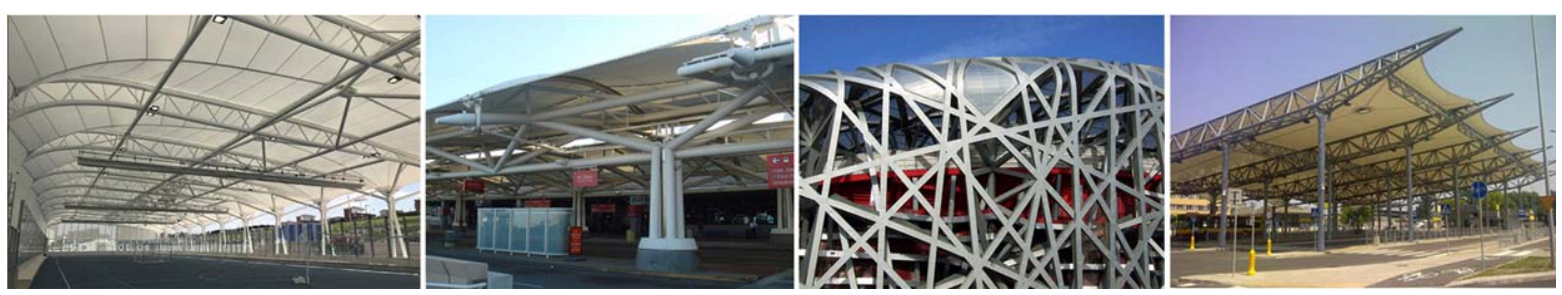

Membrane structures frequently end up being conventional steel structures.

Some recent realizations are not yet rid of this drawback but others have assumed it to the point of improving the behaviour of the structure even more reaching some successful achievements, such as spoked-wheels, cable-beams, active bending, large size inflatable structures or the Tensairity system, among others. They are based on lightness, the ability to follow the load paths and the right combination of curvature, depth and prestress. Small and medium-size tensile structures have also proved to be efficient avoiding bending, stiffness, massiveness and limiting buckling by using circular hollow steel sections improved by tapering, trussing, tying, coupling, branching or flying. Appropriateness may be assessed checking materials (properties, quantity, weight $/ \mathrm{m}^{2}$ ) , geometry (height/side length, sag/span, edge ratio, curvatures, covered surface in plan/surface of the membrane, displacements) and forces (internal forces, load paths, prestress, reaction forces, mechanical work $\operatorname{Tra}=\Sigma\left(\mathrm{P}^{*} \ell\right)$ or the ratio of mass to force path Bic $\left.=\mathrm{m} / \mathrm{Tra}\right)$. Ease of assembly, cost, appearance and elegant connection details should also be considered.

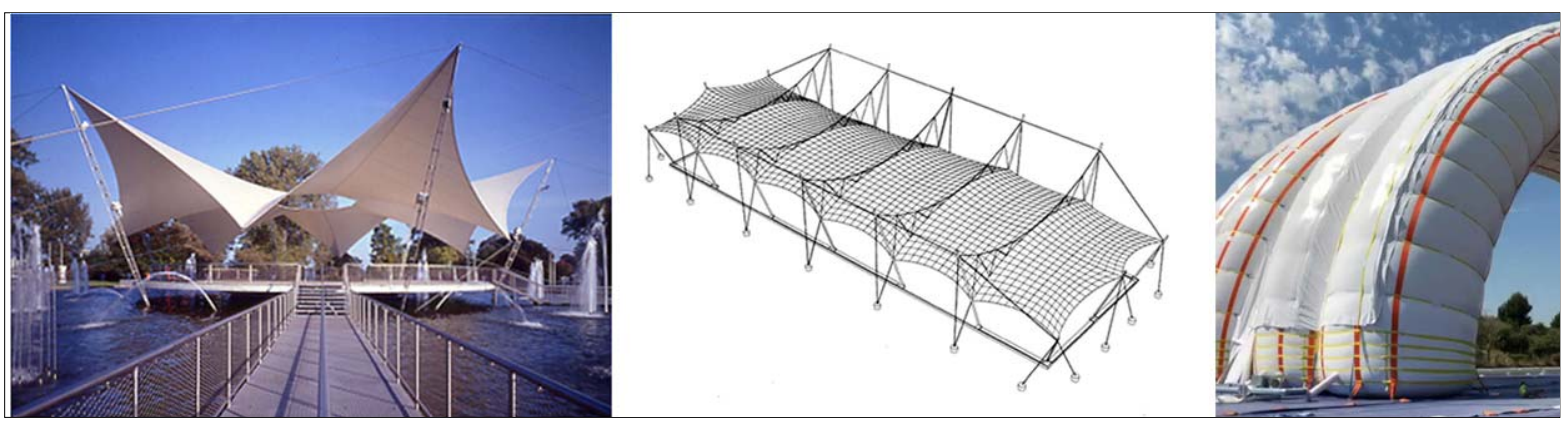

A collection of examples will illustrate what is meant by appropriateness.

\section{REFERENCES}

B.Bridgens \& M.Birchall, 2012: "Form and function". Engineering Structures, nº 44, pp. 1-12.

IL 21, 1979: "Form $\leftrightarrow$ Force $\leftrightarrow$ Mass". Institute for Lightweight Structures, Stuttgart.

J.Llorens, 2019: "Detailing masts". Structural Membranes, Barcelona, pp. 359-366. 